\title{
Bioética en salud pública: protección y confianza en VIH/SIDA
}

\author{
MIGUEL KOTTOW ${ }^{(1)}$
}

\section{RESUMEN}

La pandemia VIH/SIDA ha resistido las medidas preventivas tradicionales de la epidemiología que, además de ser ineficaces, generan actitudes sociales de discriminación y rechazo. Por esta falta de proporcionalidad entre eficacia mínima y los altos riesgos de exclusión social, se ha instaurado un excepcionalismo epidemiológico que omite medidas de control y prevención validadas para enfermedades sexualmente transmitidas. El respeto a la autonomía individual se ve tensionado en el caso de mujeres embarazadas en quienes el hallazgo de seropositividad permitiría un tratamiento que reduce la transmisión vertical de $25 \%$ a menos de $8 \%$. Esta eficaz protección de los recién nacidos ha sugerido que el serodiagnóstico de VIH/SIDA sea obligatorio durante el embarazo, una sugerencia fortalecida por la reciente disponibilidad del examen rápido, que permitiría tamizar a mujeres que acuden tardiamente al control obstétrico.

La bioética clínica, principialista e individualista, inspirada en el respeto por las personas, defiende la autonomía y rechaza toda obligatoriedad, aunque sea de beneficio para los niños y en reducir la incidencia de VIH/SIDA. En cambio, la bioética en salud pública, fundamentada en la protección, reflexiona sobre la conveniencia de limitar la autonomía individual cuando ella obstaculiza la aplicación de medidas preventivas y terapéuticas demostradamente eficaces. En la medida que la [bio]ética de protección sea una meta irrenunciable de la salud pública, logrará fortalecer la confianza y la participación ciudadana en políticas públicas.

Palabras clave: Autonomía, bioética, confianza, protección, VIH/SIDA.

\section{ABSTRACT}

\section{BIOETHICS IN PUBLIC HEALTH: PROTECTION AND ASSURANCE IN HIV/AIDS}

The HIV/AIDS pandemic has withstood traditional preventive measures of epidemiology. Such measures not only are inefficient, but have generated social attitudes of discrimination and rejection. The evident disproportion between minimal efficacy levels and the high risks of social exclusion, has built on an epidemiological exceptionalism with relation to sexually transmitted diseases, which is reflected on the resulting omission of preventive and control measures. Respect for individual autonomy is undermined in cases such as pregnant women, in whom the detection of seropositivity would enable the delivery of a therapy decreasing vertical transmission from $25 \%$ to less than $8 \%$. Such efficient protection of the newborn has resulted in the advice for the

\footnotetext{
${ }^{(1)}$ Escuela de Salud Pública. Facultad de Medicina. Universidad de Chile. Independencia 939, Santiago. Chile. kottow@terra.cl
} 
implementation of a mandatory HIV/AIDS serodiagnosis during pregnancy, a recommendation that is supported by the recent availability of a fast test, which would enable the screening of women presenting belatedly to obstetrical controls.

Clinical bioethics, a principleist and individualist discipline, is inspired on respect towards people and fosters autonomy while rejecting all compulsoriness, even if it should result in a benefit for children and to decrease the prevalence of HIVIAIDS. Bioethics in public health, however, are based on protection, considers the convenience of restrictions on individual autonomy when the latter hinders the implementation of efficiency-proven preventive and therapeutic measures. As long as protection [bio]ethics become an inalienable goal of public health, citizen assurance and participation in public policies will be leveraged.

Keywords: Autonomy, bioethics, assurance, protection, HIV/AIDS.

La salud pública chilena se abre a la bioética referida a su disciplina, en concordancia con lo que ocurre en algunas relevantes publicaciones internacionales ${ }^{1}$. Los recientemente publicados trabajos referidos a los aspectos éticos del diagnóstico rápido de $\mathrm{VIH} / \mathrm{SIDA}^{2}$, y a la confianza/desconfianza puesta en los sistemas de salud por quienes acuden a ellos ${ }^{3}-10 s$ impropiamente denominados usuarios-, ganan ambos en interés al motivar un debate que, lejos de ser crítico, pretende ratificar la importancia de estos análisis al ampliar y matizar los temas tratados.

\section{Excepcionalismo epidemiológico en VIH/SIDA}

La necesidad de enfocar la pandemia VIH/SIDA de un modo diferente a lo tradicionalmente reconocido como eficaz estrategia contra enfermedades infecciosas en general, y las sexualmente transmitidas en particular, se basa en tres percepciones: 1) La identificación de personas infectadas no logra desplegar medidas precautorias y preventivas eficientes. 2) Las terapéuticas para los seropositivos han sido un gran avance médico, pero son problemáticas en cuanto a acceso, costos, tolerancia, efectividad. 3) El diagnóstico de seropositividad al VIH no es social ni éticamente neutro, pues persisten juicios moralmente negativos y resabios de discriminación y exclusión frente a los grupos en riesgo: drogadictos, homosexuales, prostitutas, personas sexualmente promiscuas.
La baja efectividad de los programas epidemiológicos tradicionales y su potencial discriminatorio, llevaron a establecer la doctrina del excepcionalismo ${ }^{4}$ : no aplicar medidas rutinarias que en la pandemia de SIDA no son efectivas en lo sanitario, pero sí lesivas en lo social. A eso es preciso agregar la fuerte dependencia contextual de estos factores, de modo que las experiencias sanitarias y las publicaciones académicas de una realidad social no se extrapolan fácilmente a otras. Sobre todo los países que no están en la frontera del desarrollo, requieren elaborar su propia reflexión y planificar estrategias acordes con su realidad, considerando que según una revisión reciente no más del $12 \%$ de la población infectada en países subdesarrollados recibe terapia antiretroviral. Este promedio oculta las bajísimas coberturas de naciones como India (4\%) o Rusia $(3 \%)^{5}$.

Vidal destaca algunas posibles ventajas del recientemente desarrollado examen rápido frente al diagnóstico tradicional de VIH/SIDA (Elisa, Western blot), como son la oportuna inmediatez del resultado y la mayor discreción de realizarlo en privado. No queda muy claro si la privacidad queda mejor resguardada con la adquisición comercial del examen en comparación con acudir a un centro diagnóstico, pero al parecer las diferencias no son substanciales por cuanto podrían ser eliminadas con las debidas precauciones. Por otro lado, el procedimiento privado genera una brecha mayor entre diagnóstico y acceso a tratamiento, que la institucionalización de ambos momentos. Hay 
que considerar, asimismo, que todo acto médico que se privatiza es motivo de discriminación entre pudientes y desposeídos, transformado en franca inequidad si el acceso privado fuese sanitariamente más beneficioso.

Los factores mencionados, y la aparición de nuevos métodos diagnósticos, generan un excepcionalismo que es de facto, obligando a la revisión permanente de las políticas sanitarias que se implementan, o se omiten, en relación al VIH/SIDA, y su adecuación a las realidades sociales locales. El requerimiento de la bioética a la salud pública es no innovar a menos que ello implique un aumento de beneficios a los afectados o una mejor protección de la población. Mientras esta ponderación no se realice, existe el riesgo que una modificación de las medidas preventivas o del método diagnóstico pudiese resultar en un peor engranaje con las disponibilidades terapéuticas.

\section{Protección y autonomía en salud pública}

Como contraparte a lo anteriormente planteado, las políticas de salud pública deben estar atentas a no descartar posibles ventajas de una innovación. Consideraciones bioéticas han condenado tanto la aplicación obligatoria como la engañosa del diagnóstico de SIDA, respetando la autonomía de las personas para solicitar o consentir al examen. Desde hace algún tiempo se discute acaso la mujer embarazada también se ha de amparar tras el respeto a su autonomía, considerando que con tratamientos adecuados es posible bajar el riesgo de infección transplacentaria o por lactancia del $25 \%$ al $8 \%$. La eficaz reducción de transmisión vertical del VIH ha llevado a proponer el serodiagnóstico obligatorio como parte del cuidado de la mujer embarazada, pero la sugerencia enfrenta argumentos de orden médico, económico y ético ${ }^{6}$.

En regiones más apartadas, con cobertura sanitaria geográficamente desigual, suele ocurrir que las mujeres llevan su embarazo sin controles obstétricos y solo acuden al centro médico con ocasión del parto. La posibilidad de realizar un serodiagnóstico se limita al escaso tiempo que la mujer estará bajo cuidados médicos directos, en cuyo caso habría una notoria ventaja en contar con el examen rápido para instaurar de inmediato una terapia antiretroviral que no será tan eficaz como el esquema 076, pero evitará la infección de un número importante de neonatos. La sugerencia de hacer obligatoria la pesquisa de VIH/SIDA en embarazadas para proteger al hijo gana fuerza en el caso de mujeres que acuden tardíamente a centros médicos y que solo podrán ser pesquisadas con examen rápido, eventualmente sin su consentimiento, puesto que la inminencia del parto les restaría ecuanimidad de juicio.

Si la bioética en salud pública permaneciese fiel al principialismo de Georgetown y confirmase que el principio de autonomía mantiene su validez siempre que se cumplan los requisitos de intencionalidad, conocimiento y ausencia de coerción ${ }^{2}$, debería ser cautelado el derecho de la madre a negarse al serodiagnóstico, en independencia de las consecuencias epidemiológicas o del probable daño al niño. Sin embargo, va quedando suficientemente en claro que la bioética en salud pública obedece a una deliberación de otro orden, donde eventualmente la autonomía tiene que ceder frente a intereses de terceros o del bien común que están siendo cautelados por políticas sanitarias específicas y efectivas. El excepcionalismo en VIH/SIDA que defiende la autonomía por encima de programas epidemiológicos inciertos, debe ser revisado, incluso planteándose una forma de excepcionalismo bioético, donde la autonomía es limitada excepcionalmente por motivos de eficacia epidemiológica y protección del niño por nacer.

Llevado a una ponderación comparativa, la autonomía de los individuos puede y debe ser coartada allí donde ello es necesario para asegurar una protección pública impostergable. En el caso de las mujeres embarazadas, es convincente argumentar que su autonomía debe ser sobreseída en beneficio de sus hijos, haciendo impositivo el diagnóstico a condición que se ponga a disposición de las seropositivas el tratamiento completo más eficaz existente. Por analogía, el examen rápido podría ser obligatorio 
en mujeres que se presentan al parto y donde la probabilidad que se nieguen a un eventual tratamiento es menor. Lo que para la bioética principialista significaría una violación indebida de la autonomía, se constituye desde la visión de la salud pública en una restricción justificada y excepcional de la voluntad autónoma en aras de la reducción de tasas de infección vertical y protección del nasciturus, en consideración a que, según UNICEF, la mayoría de los 1000 niños que mueren al día por VIH/SIDA han sido infectados por vía vertical ${ }^{7}$.

\section{Crisis de confianza en servicios sanitarios}

La pérdida de confianza en el mundo tardomoderno ha sido motivo reciente de preocupación en las relaciones entre instituciones públicas y ciudadanía, muy especialmente en el área sanitaria. La filósofa británica $\mathrm{O}$. $\mathrm{O}^{\prime} \mathrm{Ne}$ ill dedicó sus "BBC Reith Lectures 2002" al tema de la confianza, poniendo énfasis en que la crisis no depende tanto de ver frustrados los reclamos de derechos, como del fervor con que los derechos son reconocidos y respetados: "la democracia presupone derechos y los derechos presuponen obligaciones". Una declaración de derechos humanos solo logra el compromiso social de respetarlos si se "muestra quién es requerido de hacer qué para quién, o por qué está siendo requerido de hacerlo". Así se explica que tantos derechos sean formulados en un vacío social que no los acoge. Los movimientos cívicos que claman por derechos tienen menos posibilidades de imponerse que las políticas que reconocen obligaciones.

Una razón predominante de la crisis de confianza en sociedades modernas es que las expectativas ciudadanas expresadas en forma de derechos no tienen un correlato efectivo con obligaciones estatales o sociales. Ejemplo craso de ello es la formulación de un derecho a [protección de la] salud que aparece en prácticamente todas las constituciones americanas ${ }^{10}$, pero al cual las instituciones sociales y el Estado responden en forma muy fragmentaria por cuanto no asumen una obligación sanitaria que dé respuesta al mandato constitucional. $\mathrm{La}$ reacción ciudadana es la desconfianza al no ver atendidas sus necesidades de salud/enfermedad.

El argumento de Hevia ${ }^{3}$ apunta en gran medida a lo que podría llamarse la confianza ex post: si el trato del médico fue satisfactorio, la prescripción benéfica, la duración de la consulta y el esfuerzo técnico desplegado adecuados, tanto el médico como el sistema habrán conquistado la confianza del paciente. Podría también caracterizarse la evaluación en términos de calidad de atención, detectándose ciertos resabios de paternalismo en esta interacción, ilustrado, según el autor, por la tranquilidad sentida por el paciente de haber elegido bien si se encuentra con una pared tapizada de diplomas en la consulta del médico

Otro modo de ver la confianza en relación a las acciones sanitarias es considerarla un elemento ex ante. La bioética lleva muchos años insistiendo que la relación entre paciente y médico, así como entre probando e investigador, debe sustentarse en un flujo informativo acabado que abarque todo lo disponible y pertinente para que el paciente tome una decisión clínica informada, el probando otorgue su consentimiento y, para el caso de la salud pública, el ciudadano acepte sacrificar aquella parte de su autonomía que interfiere con una efectiva política sanitaria de protección. Este esfuerzo informativo se lleva a cabo en un clima de confianza técnica donde se cuenta con la veracidad y la honestidad del informante. No obstante, aun el más depurado de los procesos informativos inevitablemente deja un residuo de incertidumbre y desconocimiento, por cuanto tanto la clínica como la salud pública son disciplinas indeterminadas e inconmensurables, donde teoría y datos empíricos se superponen solo parcialmente, en tanto que hipótesis explicativas diversas cohabitan aunque sean contradictorias.

El residuo de desinformación es imposible de resolver, por lo que paciente, probando o ciudadano deberá confiar que el terreno ignoto será manejado por el profesional con integridad moral, respetando los intereses del paciente incluso por sobre los propios. De esta manera, la confianza técnica es complementada por una confianza ética, uniendo excelencia profesional 
con preceptos bioéticos esenciales proclamados por pioneros como P. Ramsey y E. Pellegrino, quienes escribieron latamente sobre el fundamento fiduciario de las relaciones sanitarias ${ }^{11,12}$.

El camino emprendido por los servicios médicos hacia la comercialización y el viraje hacia una "nueva salud pública" que enfatiza la autoresponsabilidad, y los factores individuales determinantes del estilo de vida, han hecho lo suyo por minar la confianza en las instituciones $\mathrm{y}$ los profesionales sanitarios ${ }^{13,14}$.

La salud pública se ve principalmente afectada por la desconfianza ciudadana, no solo por la dificultad de asegurar disciplina en programas de reconocida eficacia, sino también en sus campañas por promover salud, modificar estilos de vida, delimitar autoresponsabilidades, todas basadas en una relación fiduciaria de comunicación entre expertos y la sociedad civil.

\section{Etica de protección}

De la filosofía viene la crítica que la autonomía ha tomado excesivo protagonismo tanto en la bioética clínica como en salud pública, por cuanto los individuos no pueden ni quieren ejercer total independencia de juicio en asuntos médicos ${ }^{15}$. A nivel de las relaciones interindividuales entre profesionales e investigadores biomédicos por un lado, y pacientes/probandos por el otro, reivindican y fortalecen estos argumentos la tenacidad del paternalismo y de la asimetría de poder que ocurre en estos contextos ${ }^{16}$. Más justificada parece la crítica a un exceso de autonomía en el contexto de la salud pública, pero la razón no puede ser que "algunas medidas de salud pública son compulsorias porque no es técnicamente posible introducir variaciones de gestión para acomodar preferencias individuales" ${ }^{17}$. Invirtiendo el argumento, cabe señalar que los programas de salud pública han de tener una eficacia y un valor de protección lo suficientemente alto y convincente para que la limitación de la autonomía individual sea aceptable. De este modo, se evita los abusos a la autonomía y se fomenta la confianza en una salud pública que solo actuará a la luz de evidencias de efectividad.

El nuevo examen diagnóstico rápido de VIH/SIDA presenta la ventaja de fortalecer la autonomía de las personas, concediéndoles el beneficio del mejor resguardo de su "intimidad y confidencialidad", como apunta Vidal, al no tener que acudir a un centro diagnóstico. Aflora aquí un primer momento de desconfianza, al evita el centro médico por temor a que no cautelará adecuadamente la privacidad del consultante. El costo de esta autonomía lograda por el examen rápido, es que las personas no reciben información y consejería a menos que la busquen, lo que podría no ocurrir en caso de primar la falta de confianza en las instancias terapéuticas. La deliberación bioética principialista celebrará la mayor autonomía de realizar el examen sin intervención de terceros aunque, como es inevitable, el ejercicio de la autonomía conlleve la posibilidad de decisiones erróneas. Es el acendrado acento individualista de la bioética clínica, donde el respeto por las personas tiene primacía sobre la protección.

Para la bioética en salud pública aparece aquí una herramienta diagnóstica que permite mejorar la efectividad epidemiológica frente al SIDA y de proteger ante la infección vertical a un número importante de niños, aunque ello eventualmente requiera restringir la autonomía de las mujeres y llevar a cabo el examen como rutina obligatoria. Requisito insoslayable para aceptar el predominio de la protección a costa de autonomía, es que el diagnóstico sea complementado con el acceso al mejor tratamiento médico existente. La bioética en salud pública enfatiza la protección por sobre la autonomía, ante todo para aquellos que carecen del necesario empoderamiento para ejercerla ${ }^{18}$. Con ello, la bioética pretende contribuir al fomento de una adecuada función protectora como vehículo para reforzar la confianza ciudadana en las políticas de salud pública.

\section{Conclusión}

Aunque muchas veces soslayado, sigue siendo uno de los dilemas fundamentales de la salud pública el justificar eventuales limitaciones 
a la autonomía individual cuando ciertas medidas sanitarias en pro del bien común así lo requieren. En el caso del VIH/SIDA y del diagnóstico rápido, se perfilan ventajas y desventajas personales relacionadas con la privacidad, la confidencialidad y el acceso a tratamiento cuando es necesario. Más importante es que el diagnóstico rápido es un instrumento epidemiológico que se puede aplicar a situaciones en que las pruebas tradicionales serían demasiado lentas e ineficaces. Las ventajas estratégicas del examen rápido se acrecientan al considerar que la aplicación rutinaria, eventualmente compulsoria, protegerá a un número muy significativo de niños contra la transmisión vertical del VIH. Si la bioética es fiel al postulado de proteger a los desvalidos y desmedrados, deberá sustentar una estrategia sanitaria que limita la autonomía al instituir un examen cuya obligatoriedad se justicia ampliamente por la substancial disminución de niños afectados por la transmisión vertical el VIH.

\section{REFERENCIAS}

1. VERA A. Descripción de publicaciones en bioética en revistas de salud pública. Enviado a publicación

2. VIDAL M. Cuestiones éticas y legales del uso de pruebas rápidas de diagnóstico para VIH/SIDA domiciliarias. Rev. Chil. Salud Pública 2006; 10: 99106

3. HEVIA F. Confianza y desconfianza en el sistema público de salud en México. Rev Chil Salud Pública 2006; 10: 107-111.

4. BURR C. The AIDS exception: privacy vs. Public health. En Beauchamp DE \& Streinbock B. New ethics for the public's health. New York / Oxford, Oxford Univ. Press 1999: 211-224.

5. MCGOUGH JM, REYNOLDS SJ, QUINN TC \& ZENILMAN JM. Which patients first? Setting priorities for antiretroviral therapy where resources are limited. Am. J. Public Health 2005; 95: 1173-1180.

6. CLARK PA. Mother-to-child transmission of HIV in Botswana: an ethical perspective on mandatory testing. Developing World Bioethics 2006; 6: 1-12.
7. EL MERCURIO, 19.01.07

8. O'NEILL O. A question of trust. Cambridge, Cambridge Univ. Press 2002: pág. 31.

9. Ibid.: pág. 28.

10. FUENZALIDA-PUELMA H. \& CONNOR SS. El derecho a la salud en las Américas. Washington, Organización Panamericana de la Salud 1989.

11. CARSON RA. Paul Ramsey's ethic of covenant fidelity. EnVaux KL, Vaux S \& Stenberg M. (eds.): Covenants of life. Dordrecht, Boston, London Kluwer Academic Publishers 2002: 9-18.

12. PELLEGRINO E. Toward a reconstruction of medical morality: the primacy of the act of profession and the fact of illness. The Journal of Medicine and Philosophy 1979; 4: 32-56.

13. BEAGLEHOLE R. \& BONITA R. Public health at the crossroads. Cambridge, Cambridge Univ. Press 1997.

14. PETERSEN A. \& LUPTON D. The new public health London, Thousand Oaks, New Delhi, Sage 2000.

15. O'NEILL O.Public health or clinical ethics: thinking beyond borders. Ethics and International Affairs 2002; 16: $35-45$

16. KOTTOW M. The battering of informed consent. J. Med. Ethics 2004; 30:565-569

17. O'NEILL 2002: pág. 38.

18. KOTTOW M. Etica de protección. Bogotá, Univ. Autónoma de Colombia 2006.

Recepción: 21 ene. 2007 Aprobación: 08 mar. 2007

Usted puede comentar éste y otros artículos publicados en la Revista Chilena de Salud Pública, enviando un correo electrónico a revistasp@med.uchile.cl 